\title{
Null Controllability of a Nonlinear Dissipative System and Application to the Detection of the Incomplete Parameter for a Nonlinear Population Dynamics Model
}

\author{
Yacouba Simporé ${ }^{1}$ and Oumar Traoré ${ }^{2}$ \\ ${ }^{1}$ LAMI, Universite de Ouagadougou, 01 BP 7021, Ouaga 01, Burkina Faso \\ ${ }^{2}$ LAMI, Universite de Ouagadougou, 12 BP 417, Ouaga 12, Burkina Faso \\ Correspondence should be addressed to Oumar Traoré; otraoret@gmail.com \\ Received 6 November 2015; Accepted 18 January 2016 \\ Academic Editor: Irena Lasiecka
}

Copyright ( $) 2016$ Y. Simporé and O. Traoré. This is an open access article distributed under the Creative Commons Attribution License, which permits unrestricted use, distribution, and reproduction in any medium, provided the original work is properly cited.

We first prove a null controllability result for a nonlinear system derived from a nonlinear population dynamics model. In order to tackle the controllability problem we use an adapted Carleman inequality. Next we consider the nonlinear population dynamics model with a source term called the pollution term. In order to obtain information on the pollution term we use the method of sentinel.

\section{Introduction and Main Result}

For a given real function $G$, in this paper we consider the following nonlinear system:

$$
\begin{aligned}
-\frac{\partial q}{\partial t}-\frac{\partial q}{\partial a}-\Delta q+\mu q= & \beta q(t, 0, x) G\left(\int_{0}^{A} \beta q d a\right) \\
& +\chi_{\Theta} h+\chi_{\omega} v \\
& \text { in } Q=(0, T) \times(0, A) \times \Omega, \\
q(t, a, x)=0 & \text { on } \Sigma \\
q(t, A, x)=0 & \text { in }(0, T) \times(0, A) \\
q(T, a, x)=0 & \text { in }(0, A) \times \Omega,
\end{aligned}
$$

where $\Omega$ is a bounded open subset of $\mathbb{R}^{N}, N \geq 1$, with a smooth boundary $\partial \Omega$. Let $T$ be a positive real and let $\omega, \Theta$ be two open subsets of $\Omega$ such that $\bar{\omega} \subset \Omega$ and $\Theta \subset \Omega$. Here $\chi_{\omega}, \chi_{\Theta}$ are the characteristic function of $\omega$ and $\Theta, \Delta$ is the Laplacian with respect to the spatial variable, and $\beta(a)$ and $\mu(a)$ denote, respectively, the natural fertility and the natural death rate of individuals of age $a$.
The purpose of this paper is to prove a null controllability result for (1) at any time $T$. This means more precisely that we prove here the existence of a control $v \in L^{2}\left(Q_{\omega}\right)$ such that the associated solution of (1) verifies $q(0, a, x)=0$ a.e. in $(0, A) \times$ $\Omega$. hold:

For the sequel we assume that the following assumptions

$$
\mu(a) \geq 0
$$

$$
\begin{aligned}
\lim _{a \rightarrow A} \int_{0}^{a} \mu(s) d s & =+\infty, \quad \mu \in L_{\mathrm{loc}}^{1}(0, A), \\
\beta & \in C^{2} \quad([0, A]) \\
\beta(a) & \geq 0 \quad \text { in }[0, A] \\
\exists 0 & <a_{0}<a_{1}<A \\
\text { such that } \beta(a) & =0 \quad \text { in }\left(0, a_{0}\right) \cup\left(a_{1}, A\right) \\
G & \in L^{\infty}(\mathbb{R}) .
\end{aligned}
$$

We set $Q_{\omega}=(0, T) \times(0, A) \times \omega$ and $Q_{\Theta}=(0, T) \times(0, A) \times \Theta$.

Let us now present the main results of this paper. 
For this aim we introduce the weight

$$
e_{M}(t, a)=\exp \left(\frac{M}{a t(A-a)(T-t)}\right)
$$

and define the Hilbert space

$$
L^{2}\left(e_{M}\right)=\left\{f \in L^{2}(Q): \int_{Q} f^{2} e_{M} d x d a d t<\infty\right\},
$$

endowed with the natural norm. $M$ is a real that will be defined in the following.

We have the following.

Theorem 1. Let $h \in L^{2}\left(e_{M}\right)$; then under the assumptions $\left(H_{1}\right)$, $\left(H_{2}\right)$, and $\left(H_{3}\right)$ there exists a control $v \in L^{2}\left(Q_{\omega}\right)$ satisfying $q(0, a, x)=0$ a.e. in $(0, A) \times \Omega$.

The rest of this paper is as follows: the proof of the main result is given in Section 2; in Section 3, we describe the method of sentinel; and Section 4 is devoted to the information provided by the sentinel.

\section{Proof of the Null Controllability of the Nonlinear System}

2.1. An Adapted Observability Inequality. We recall here that there exists a function $\Psi \in C^{2}(\bar{\Omega})$ such that $\Psi(x)=0$, for all $x \in \partial \Omega ; \Psi(x)>0$ for all $x \in \Omega$ and $\nabla \Psi \neq 0$ for all $\overline{\Omega-\widetilde{\omega}}$, where $\widetilde{\omega}$ is an open set such that $\overline{\widetilde{\omega}} \subset \omega \subset \Omega$. See [1].

Now we consider the following system:

$$
\begin{aligned}
\frac{\partial w}{\partial t}+\frac{\partial w}{\partial a}-\Delta w+\mu w & =f \quad \text { in } Q \\
w & =0 \quad \text { on } \Sigma \\
w(0, a, x) & =w^{0}(a, x) \quad \text { in }(0, A) \times \Omega \\
w(t, 0, x) & =g(t, x) \quad \text { in }(0, T) \times \Omega .
\end{aligned}
$$

Proposition 2. There exist positive constants $\alpha_{1}>1$ and $\lambda_{1}>$ 1 and there exists a positive constant $C(A, T)$ which depends on $A$ and $T$ such that, for $\alpha \geq \alpha_{1}, \lambda \geq \lambda_{1}$, and for all solution of (4) the following inequality holds:

$$
\begin{aligned}
& \alpha^{3} \lambda^{4} \int_{Q} e^{-2 \alpha \eta} p^{3}|w|^{2} d x d a d t \\
& \leq C \int_{Q} e^{-2 \alpha \eta} f^{2} d x d a d t \\
& \quad+C \alpha^{3} \lambda^{4} \int_{Q_{\omega}} e^{-2 \alpha \eta} p^{3}|w|^{2} d x d a d t
\end{aligned}
$$

with

$$
\begin{aligned}
& \eta(t, a, x)=\frac{e^{2 \lambda\|\Psi\|_{\infty}}-e^{\lambda \Psi(x)}}{a t(T-t)(A-a)}, \\
& p(t, a, x)=\frac{e^{\lambda \Psi(x)}}{a t(T-t)(A-a)} .
\end{aligned}
$$

For the proof of this proposition see [2].

For $f=0$ we obtain an Observability Inequality.
Lemma 3. There exist $M>0$ and $C>0$ such that the following inequality is true:

$$
\begin{aligned}
& \int_{Q} \exp \left(\frac{-M}{a t(T-t)(A-a)}\right) w^{2} d x d a d t \\
& \quad \leq C \int_{Q_{\omega}} w^{2} d x d a d t
\end{aligned}
$$

herein $w$ is the solution of system (4).

Proof. If $f=0$, (5) become

$$
\begin{aligned}
& \int_{Q} e^{-2 \alpha \eta} p^{3}|w|^{2} d x d a d t \\
& \quad \leq C \int_{Q_{\omega}} e^{-2 \alpha \eta} p^{3}|w|^{2} d x d a d t .
\end{aligned}
$$

Choosing $\lambda=\lambda_{1}$ and $\alpha=\alpha_{0} \geq \alpha_{1}$ such that $e^{-2 \alpha_{0} \eta} p^{3} \leq 1$, we obtain the result with $M=2 \alpha_{0} \exp \left(2 \lambda_{1}\|\Psi\|_{\infty}\right)$.

In the following we consider the linear system:

$$
\begin{aligned}
&-\frac{\partial q}{\partial t}-\frac{\partial q}{\partial a}-\Delta q+\mu q=\beta q(t, 0, x) b(t, x)+\chi_{\Theta} h \\
&+\chi_{\omega} v \quad \text { in } Q \\
& q(t, a, x)=0 \quad \text { on } \Sigma \\
& q(t, A, x)=0 \quad \text { in }(0, T) \times(0, A) \\
& q(T, a, x)=0 \quad \text { in }(0, A) \times \Omega, \\
& \frac{\partial \varphi}{\partial t}+\frac{\partial \varphi}{\partial a}-\Delta \varphi+\mu \varphi=0 \quad \text { in } Q \text { on } \Sigma \\
& \varphi(0, a, x)=\varphi^{0} \text { in }(0, A) \times \Omega \\
& \varphi(t, 0, x)=\int_{0}^{A} \beta \varphi b(t, x) d a
\end{aligned}
$$

in $(0, T) \times \Omega$,

where $a(t, x)$ and $b(t, x)$ are in $L^{\infty}((0, T) \times \Omega)$. Each system (9)-(10) is well posed and admits a unique solution. See [3] for the proof of existence and uniqueness of the solution.

Remark 4. For the linear case, that is, $G(x)=x$ can use the Hahn-Banach theorem for proving the existence of a control $v$ which verifies $\|q(0, \cdot, \cdot)\|_{L^{2}((0, A) \times \Omega)} \leq \epsilon$, one writes

$$
R(0)=\left\{q(0, a, x) ; v \in L^{2}\left(Q_{\omega}\right) \text { when } q \text { solves }(9)\right\} .
$$

It is easy to see that $R(0)$ is a nonempty subspace of $L^{2}((0, A) \times$ $\Omega$ ) and that

$$
R^{\perp}(0) \text { is dense in } L^{2}((0, A) \times \Omega) .
$$


It suffices to show that

$$
R^{\perp}(0) \subset\{0\} .
$$

Suppose that

$$
\varphi^{0} \in R^{\perp}\{0\}
$$

Multiplying (9) by $\varphi$ and integrating by parts, we have

$$
\begin{aligned}
\int_{Q}\left(\chi_{\Theta} h+\chi_{\omega} v\right) \varphi d x d a d t \\
=\int_{0}^{A} \int_{\Omega} q(0, a, x) \varphi^{0}(a, x) d a d x .
\end{aligned}
$$

Since

$$
\varphi^{0} \in R^{\perp}\{0\}
$$

then

$$
\int_{0}^{A} \int_{\Omega} q(0, a, x) \varphi^{0} d a d x=0 .
$$

This implies that

$$
\int_{Q}\left(\chi_{\Theta} h+\chi_{\omega} v\right) \varphi d x d a d t=0
$$

for every $v \in L^{2}\left(Q_{\omega}\right)$.

Then $\varphi=0$ on $(\Theta \cap \omega) \times(0, T) \times(0, A)$. The unique continuation property of $\varphi$ gives

$$
\varphi=0 \quad \text { on }(0, T) \times(0, A) \times \Omega .
$$

Therefore $\varphi^{0}=0$. So $R^{\perp}\{0\} \subset\{0\}$.

However, this method cannot allow the study of the nonlinear case.

2.2. The Approached Controllability of the Linear System. Now, for $\delta>0$ we consider the functional $J_{\delta}$ defined by

$$
\begin{aligned}
J_{\delta}(v)= & \frac{1}{2} \int_{Q_{\omega}}|v|^{2} d x d a d t \\
& +\frac{1}{\delta} \int_{0}^{A} \int_{\Omega} q^{2}(0, a, x) d x d a,
\end{aligned}
$$

where $q$ solves

$$
\begin{aligned}
-\frac{\partial q}{\partial t}-\frac{\partial q}{\partial a}-\Delta q+\mu q= & \beta q(t, 0, x) b(t, x)+\chi_{\Theta} h \\
& +\chi_{\omega} v \quad \text { in } Q \\
q(t, a, x)=0 & \text { on } \Sigma \\
q(t, A, x)=0 & \text { in }(0, T) \times \Omega \\
q(T, a, x)=0 & \text { in }(0, A) \times \Omega .
\end{aligned}
$$

Proposition 5. The functional $J_{\delta}$ is continuous, strictly convex, and coercive. Therefore, $J_{\delta}$ reaches its minimum at a point $v_{\delta} \in$ $L^{2}\left(Q_{\omega}\right)$. Moreover, setting $q_{\delta}$ the associated solution of $(21)$ and $\varphi_{\delta}$ the solution of (10) with $\varphi_{\delta}(0, a, x)=-(1 / \delta) q_{\delta}(0, a, x)$ has $\chi_{\omega} v_{\delta}=\chi_{\omega} \varphi_{\delta}$ and there exist $C>0, K_{0}>0$ independent of $\delta$, such that

$$
\begin{gathered}
\int_{q_{\omega}}\left|\varphi_{\delta}\right|^{2} d x d a d t \leq C\|h\|_{L^{2}\left(e_{M}\right)}^{2}, \\
\int_{0}^{A} \int_{\Omega} q_{\delta}^{2}(0, a, x) d x d a \leq \delta K_{0}\|h\|_{L^{2}\left(e_{M}\right)}^{2} .
\end{gathered}
$$

Remark 6. As we will see Proposition 5 solves the problem of existence of the approached sentinel for the linear case.

Proof. It is easy to check that $J_{\delta}$ is coercive, continuous, and strictly convex. Then it admits a unique minimizer $v_{\delta}$. The maximum principle gives that $\chi_{\omega} v_{\delta}=\chi_{\omega} \varphi_{\delta}$.

Multiplying (21) with $v=v_{\delta}$ by $\varphi_{\delta}$ and integrating by parts, we obtain

$$
\begin{aligned}
- & \frac{1}{\delta} \int_{0}^{A} \int_{\Omega} q_{\delta}^{2}(0, a, x) d x d a \\
& =\int_{Q_{\Theta}} h \varphi_{\delta} d x d a d t+\int_{Q_{\omega}} v_{\delta} \varphi_{\delta} d x d a d t .
\end{aligned}
$$

So

$$
\begin{aligned}
& \frac{1}{\delta} \int_{0}^{A} \int_{\Omega} q_{\delta}^{2}(0, a, x) d x d a+\int_{Q_{\omega}} \varphi_{\delta}^{2} d t d a d x \\
& \quad \leq \int_{Q_{\Theta}}\left|h \varphi_{\delta}\right| d x d a d t
\end{aligned}
$$

Using the inequality of Young, we obtain for any $R>0$

$$
\begin{aligned}
\frac{1}{\delta} \int_{0}^{A} \int_{\Omega} q_{\delta}^{2}(0, a, x) d x d a+\int_{\mathrm{Q}_{\omega}} \varphi_{\delta}^{2} d t d a d x \\
\leq 2 R\|h\|_{L^{2}\left(e_{M}\right)}^{2} \\
\quad+\frac{1}{2 R} \int_{Q} \exp \left(\frac{-M}{a t(T-t)(A-a)}\right) \varphi_{\delta}^{2} d x d a d t
\end{aligned}
$$

Now, inequalities (7) and (25) give

$$
\begin{aligned}
& \frac{1}{\delta} \int_{0}^{A} \int_{\Omega} q_{\delta}^{2}(0, a, x) d x d a+\int_{Q_{\omega}} \varphi_{\delta}^{2} d x d a d t \\
& \quad \leq 2 R\|h\|_{L^{2}\left(e_{M}\right)}^{2}+\frac{C}{2 R} \int_{Q_{\omega}} \varphi_{\delta}^{2} d x d a d t
\end{aligned}
$$

Choosing $R$ such that $2 R=C$ we have

$$
\begin{aligned}
& \frac{1}{\delta} \int_{0}^{A} \int_{\Omega} q_{\delta}^{2}(0, a, x) d x d a+\frac{1}{2} \int_{Q_{\omega}} \varphi_{\delta}^{2} d x d a d t \\
& \quad \leq 2 R\|h\|_{L^{2}\left(e_{M}\right)}^{2} .
\end{aligned}
$$


We conclude that

$$
\begin{gathered}
\int_{Q_{\omega}}\left|\varphi_{\delta}\right|^{2} d t d a d x \leq 2 R\|h\|_{L^{2}\left(e_{M}\right)}^{2}, \\
\int_{0}^{A} \int_{\Omega} q_{\delta}^{2}(0, a, x) d x d a \leq 2 R \delta\|h\|_{L^{2}\left(e_{M}\right)}^{2} .
\end{gathered}
$$

Proposition 7. The function $q_{\delta}$ solution of system (21) verifies

$$
\left\|q_{\delta}\right\|_{L^{2}\left((0, T) \times(0, A) ; H^{1}(\Omega)\right)} \leq C\left(\|h\|_{L^{2}\left(e_{M}\right)}+\|h\|_{L^{2}(Q)}\right) .
$$

Proof. Let $\lambda_{0}$ be a positive constant. Put $\widehat{q}_{\delta}=e^{-\lambda_{0} t} q_{\delta}$. Then, $\hat{q}_{\delta}$ solves

$$
\begin{aligned}
& -\frac{\partial \widehat{q}_{\delta}}{\partial t}-\frac{\partial \widehat{q}_{\delta}}{\partial a}-\Delta \widehat{q}_{\delta}+\left(\mu+\lambda_{0}\right) \hat{q}_{\delta} \\
& \quad=\beta \widehat{q}_{\delta}(t, 0, x) b(t, x)+\chi_{\Theta} e^{-\lambda_{0} t} h+\chi_{\omega} e^{-\lambda_{0} t} \varphi_{\delta}
\end{aligned}
$$

in $Q$

$$
\begin{array}{ll}
\widehat{q}_{\delta}(t, a, x)=0 & \text { on } \Sigma \\
\widehat{q}_{\delta}(t, A, x)=0 & \text { in }(0, T) \times \Omega \\
\widehat{q}_{\delta}(T, a, x)=0 & \text { in }(0, A) \times \Omega .
\end{array}
$$

Multiplying (30) by $\widehat{q}_{\delta}$ and integrating by parts, we obtain

$$
\begin{aligned}
& \frac{1}{2} \int_{0}^{A} \int_{\Omega} \widehat{q}_{\delta}^{2}(0, a, x) d x d a+\frac{1}{2} \int_{0}^{T} \int_{\Omega} \widehat{q}_{\delta}^{2}(t, 0, x) d x d t \\
& \quad+\int_{Q}\left|\nabla \widehat{q}_{\delta}\right|^{2} d x d a d t+\lambda_{0}\left\|\widehat{q}_{\delta}\right\|_{L^{2}(\mathrm{Q})}^{2} \\
& \leq \frac{1}{2} \int_{0}^{T} \int_{\Omega} \widehat{q}_{\delta}^{2}(t, 0, x) d x d t \\
& \quad+A\|\beta\|_{\infty}^{2}\|b\|_{\infty}^{2}\left\|\hat{q}_{\delta}\right\|_{L^{2}(\mathrm{Q})}^{2}+\|h\|_{L^{2}(\mathrm{Q})}^{2} \\
& \quad+C\left\|\hat{q}_{\delta}\right\|_{L^{2}(\mathrm{Q})}^{2}+2 R\|h\|_{L^{2}\left(e_{M}\right)}^{2} .
\end{aligned}
$$

Choosing $\lambda_{0}>A\|\beta\|_{\infty}^{2}\|b\|_{\infty}^{2}+C+1$, we obtain

$$
\left\|\nabla \widehat{q}_{\delta}\right\|_{L^{2}(Q)}+\left\|\widehat{q}_{\delta}\right\|_{L^{2}(Q)} \leq C\left(\|h\|_{L^{2}(Q)}+\|h\|_{L^{2}\left(e_{M}\right)}\right) .
$$

This gives the desired estimation.

2.3. Study of the Nonlinear Case. In the following we set

$$
b(t, x)=G(\eta)
$$

Let $\Lambda$ be an operator defined on $L^{2}((0, T) \times \Omega)$ by

$$
\Lambda(\eta)=\int_{0}^{A} \beta(a) q_{\delta}(\eta) d a,
$$

where $q_{\delta}(\eta)$ is the solution of the following system:

$$
\begin{aligned}
& -\frac{\partial q_{\delta}}{\partial t}-\frac{\partial q_{\delta}}{\partial a}-\Delta q_{\delta}+\mu q_{\delta} \\
& \quad=\beta q_{\delta}(t, 0, x) G(\eta)+\chi_{\Theta} h+\chi_{\omega} \varphi_{\delta} \quad \text { in } Q \\
& q_{\delta}(t, a, x)=0 \quad \text { on } \Sigma \\
& q_{\delta}(t, A, x)=0 \quad \text { in }(0, T) \times(0, A) \\
& q_{\delta}(T, a, x)=0 \quad \text { in }(0, A) \times \Omega .
\end{aligned}
$$

Then we have the following.

Proposition 8. The operator $\Lambda$ is continuous, bounded, and compact of $L^{2}((0, T) \times \Omega)$. Then $\Lambda$ admits a fixed point.

Proof. The proof will be done in two steps as follows.

Step 1 (boundedness and compactness of $\Lambda$ ). Let

$$
Y(t, a, x)=\int_{0}^{A} \beta(a) q_{\delta} d a,
$$

and denote $(0, T) \times \Omega=Q_{T}$.

Then $Y$ is the solution of the following system:

$$
\begin{aligned}
\frac{\partial Y(\eta)}{\partial t}-\Delta Y(\eta)+\int_{0}^{A} \mu \beta(a) q_{\delta} d a & =Z(\eta) \quad \text { in } Q_{T} \\
Y(\eta)(t, x) & =0 \\
& \text { on }(0, T) \times \partial \Omega \\
Y(\eta)(0, x)= & 0 \text { in } \Omega
\end{aligned}
$$

with

$$
\begin{aligned}
& Z(\eta) \\
& =\int_{0}^{A}\left(\beta_{a}^{\prime} q_{\delta}+\chi_{\omega} \beta \varphi_{\delta}+\beta^{2} q_{\delta}(t, 0, x) G(\eta)+\beta h\right) d a .
\end{aligned}
$$

Under assumptions $\left(H_{1}\right),\left(H_{2}\right)$, and $\left(H_{3}\right)$ and the results and owing to the estimation on $q_{\delta}$ and $\varphi_{\delta}$ the function $Z(\eta)$ satisfies

$$
\begin{aligned}
& \|Z(\eta)\|_{L^{2}((0, T) \times \Omega)} \\
& \quad \leq C\left(\left\|\beta_{a}^{\prime}\right\|_{\infty},\|\beta\|_{\infty},\|G\|_{\infty}\right)\left(\|h\|_{L^{2}\left(e_{M}\right)}+\|h\|_{L^{2}(Q)}\right) .
\end{aligned}
$$

System (37) is a heat equation where the source term and the initial condition are bounded, respectively, in $L^{2}\left(Q_{T}\right)$ and $L^{2}(\Omega)$.

Then $Y(\eta)$ is bounded in $L^{2}\left(0, T ; H_{0}^{1}(\Omega)\right)$ and $\partial Y(\eta) / \partial t$ is bounded in $L^{2}\left(0, T ; H_{0}^{-1}(\Omega)\right)$. Hence, using the LionsAubin lemma we conclude that $\Lambda$ is bounded and compact in $L^{2}\left(Q_{T}\right)$. 
Step 2 (continuity of $\Lambda$ ). Let $\eta_{n} \rightarrow \eta$ strongly in $L^{2}\left(Q_{T}\right)$. Then we can show that the sequence $Y\left(\eta_{n}\right)$ converges to $Y(\eta)$ strongly. For all $\eta_{n} \in \mathbb{R}, q_{\delta}\left(\eta_{n}\right)$ and $\varphi_{\delta}\left(\eta_{n}\right)$ are bounded independently to $\eta_{n}$. Therefore $Z\left(\eta_{n}\right)$ is bounded in $L^{2}\left(Q_{T}\right)$. Then we can extract a subsequence $X\left(\eta_{n_{k}}\right)$ such that

$$
\begin{aligned}
Y_{\eta_{k}} & \longrightarrow Y(\eta) \quad \text { on } Q_{T} \\
Z_{\eta_{k}} & \longrightarrow Z(\eta) \quad \text { weakly in } Q_{T} \\
\int_{0}^{A} \mu \beta q_{\delta}\left(\eta_{n}\right) d a & \longrightarrow \int_{0}^{A} \mu \beta q_{\delta}(\eta) d a
\end{aligned}
$$

weakly in $L^{2}\left(Q_{T}\right)$.

Then $Y(\eta)$ is a solution of (37). We deduce that the sequence $\left(Y\left(\eta_{n}\right)\right)$ converges to $Y(\eta)$.

Since the operator $\Lambda$ is continuous, bounded, and compact on $L^{2}\left(Q_{T}\right)$ onto $L^{2}\left(Q_{T}\right)$, Schauder's fixed-point theorem (see $[4,5]$ ) implies that $\Lambda$ admits a fixed point.

There exists $\eta \in L^{2}\left(Q_{T}\right)$ such that

$$
\Lambda(\eta)=\eta=\int_{0}^{A} \beta q_{\delta} d a
$$

Then $q_{\delta}$ solves

$$
\begin{aligned}
& -\frac{\partial q_{\delta}}{\partial t}-\frac{\partial q_{\delta}}{\partial a}-\Delta q_{\delta}+\mu q_{\delta} \\
& \quad=\beta q_{\delta}(t, 0, x) G\left(\int_{0}^{A} \beta q_{\delta} d a\right)+\chi_{\Theta} h+\chi_{\omega} \varphi_{\delta}
\end{aligned}
$$

in $Q$

$$
\begin{array}{ll}
q_{\delta}(t, a, x)=0 & \text { on } \Sigma \\
q_{\delta}(t, A, x)=0 & \text { in }(0, T) \times(0, A) \\
q_{\delta}(T, a, x)=0 & \text { in }(0, A) \times \Omega .
\end{array}
$$

From the foregoing, we have that $\varphi_{\delta}$ and $q_{\delta}$ are bounded in $L^{2}(Q)$.

Then we can extract a subsequence of $\left(q_{\delta}\right)$ still denoted by $\left(q_{\delta n}\right)$, such that

$$
\begin{aligned}
q_{\delta} & \rightarrow q \text { in } L^{2}(Q), \\
\int_{0}^{A} \beta q_{\delta} d a & \longrightarrow \int_{0}^{A} \beta q d a \quad \text { in } L^{2}\left(Q_{T}\right) .
\end{aligned}
$$

By the same idea

$$
\chi_{\omega} \varphi_{\delta} \rightarrow \chi_{\omega} v \quad \text { in } L^{2}(Q) .
$$

Since $\int_{0}^{A} \beta q_{\delta} d a \rightarrow \int_{0}^{A} \beta q d a$, then there exists a subsequence still denoted by $\int_{0}^{A} \beta q_{\delta} d a$ such that $\int_{0}^{A} \beta q_{\delta} d a \rightarrow \int_{0}^{A} \beta q d a$ a.e in $Q_{T}$.

Now since $G$ is continuous, then

$$
G\left(\int_{0}^{A} \beta q_{\delta} d a\right) \rightarrow G\left(\int_{0}^{A} \beta q d a\right) \text { a.e in } Q_{T} \text {. }
$$

Therefore, one derives that $q$ solves the following system:

$$
\begin{aligned}
&-\frac{\partial q}{\partial t}-\frac{\partial q}{\partial a}-\Delta q+\mu q= \beta q(t, 0, x) G\left(\int_{0}^{A} \beta q d a\right) \\
&+\chi_{\Theta} h+\chi_{\omega} v \quad \text { in } Q \\
& q(t, a, x)=0 \quad \text { on } \Sigma \\
& q(t, A, x)=0 \quad \text { in }(0, T) \times(0, A) \\
& q(T, a, x)=0 \quad \text { in }(0, A) \times \Omega,
\end{aligned}
$$

and we have also, for $\delta \rightarrow 0, q(0, a, x)=0$ a.e. in $(0, A) \times$ $\Omega$.

\section{Application to the Detection of the Incomplete Parameter for a Nonlinear Population Dynamics Model}

For a given positive real function $F$, we consider the following nonlinear population dynamics model:

$$
\begin{aligned}
& \frac{\partial y}{\partial t}+\frac{\partial y}{\partial a}-\Delta y+\mu y=\lambda \hat{\xi} \quad \text { in } Q \\
& y(t, a, x)=0 \quad \text { on } \Sigma \\
& y(t, 0, x)=F\left(\int_{0}^{A} \beta(a) y(\lambda, \tau) d a\right) \\
& \text { in }(0, T) \times \Omega \\
& y(0, a, x)=y^{0}(a, x)+\tau \hat{y}^{0}(a, x) \\
& \text { in }(0, A) \times \Omega,
\end{aligned}
$$

where $\Omega$ is a bounded open subset of $\mathbb{R}^{N}, N \geq 1$, with a smooth boundary $\partial \Omega$. Let $T$ be a positive real and let $\omega$ be an open subset such that $\bar{\omega} \subset \Omega$. Here, $y(t, a, x)$ is the distribution of individuals of age $a$ at time $t$ and location $x \in \Omega, \chi_{\omega}$ is the characteristic function of $\omega, A$ is the maximal live expectancy, $\Delta$ is the Laplacian with respect to the spatial variable, and $\beta(a)$ and $\mu(a)$ denote, respectively, the natural fertility and the natural death rate of individuals of age $a$. Thus, the formula $\int_{0}^{A} \beta(a) y(t, a, x) d a$ denotes the distribution of newborn individuals at time $t$ and location $x$. In an oviparus species it denotes the total eggs at time $t$ and position $x$. Therefore, the quantity $F\left(\int_{0}^{A} \beta(a) y(t, a, x) d a\right)$ is the distribution of eggs that hatch at time $t$ and position $x$.

System (47) describes the evolution of internal controlled age and space structured population under inhospitable boundary conditions in the case that the flux of individuals has the form $-\nabla y(t, a, x)$.

In system (47) $y^{0}$ is given in $L^{2}((0, A) \times \Omega)$.

The data of system (47) are incomplete in the following sense:

(i) $\hat{y}^{0} \in L^{2}((0, A) \times \Omega)$ is unknown and $\left\|\hat{y}^{0}\right\|_{L^{2}((0, A) \times \Omega)}=$ 1 ; 
(ii) $\widehat{\xi} \in L^{2}(Q)$ is unknown and $\|\widehat{\xi}\|_{L^{2}(Q)}=1$;

(iii) $\lambda \in \mathbb{R}$ is unknown and small enough;

(iv) $\tau \in \mathbb{R}$ is unknown and small enough.

We denote by $y_{\mathrm{obs}}=m_{0}$ the observation that is a function in $L^{2}\left(Q_{\Theta}\right)$, where $\Theta \subset \Omega$ is the observation set. We know that (47) has a unique solution that we denote by $y(\lambda, \tau)=$ $y(t, s, x, \lambda, \tau)$ in some relevant space. The question is as follows:

How to calculate the incomplete term $\lambda \widehat{\xi}$ independently from the variation $\tau \widehat{y}^{0}$ around the initial data?

The question above is natural and leads to some developments. An answer is given by the least squares method. This method consists in considering the unknowns $\left\{\lambda \xi, \tau y_{0}\right\}=$ $\{\nu, \vartheta\}$, as control variables; then the state $y(t, s, x, \nu, \vartheta)$ has to be driven as close as possible to $m_{0}$. So one studies an optimal control problem. By this way one looks to the pair $\{\nu, \vartheta\}$ but unfortunately there is possibility to find $\nu$ or $\vartheta$ independently. The sentinel method of Lions [6] is a particular least squares method which is adapted to the identification of parameters in ecosystems with incomplete data. Many papers are devoted to this topic in the literature. The sentinel concept relies on the following three objects: some state equation (e.g., (47)), some observation function $\left(y_{\mathrm{obs}}\right)$, and some control function $v$ to be determined. Many papers use the definition of Lions in the theoretical aspect (see, e.g., Bodart [7], Bodart and Fabre $[8,9]$ ), as well as in the numerical one (see Bodart and Demeestere [10], Demeestere [11], and Kernevez [12]).

In [13] the author studies the detection of incomplete parameter for a linear population dynamic model. In this paper we are concerned by the nonlinear case. Moreover our method allows us to easily address the numerical aspect.

We suppose that $F \in C^{1}(\mathbb{R})$ and $F^{\prime} \in L^{\infty}(\mathbb{R})$.

Proposition 9. The functions $\lambda \mapsto y(\lambda, \tau)$ and $\tau \mapsto y(\lambda, \tau)$ are differentiable at the point 0.

Proof. Let

$$
\widehat{y}(t, a, x)=e^{-\lambda_{0} t}\left(y(\lambda, \tau)-y_{0}\right)
$$

where $y(\lambda, 0)=y_{0}$.

It is easy to prove that $\hat{y}$ is the solution of the following system:

$$
\begin{aligned}
& \frac{\partial \hat{y}}{\partial t}+\frac{\partial \hat{y}}{\partial a}-\Delta \widehat{y}+\left(\mu+\lambda_{0}\right) \hat{y}=0 \quad \text { in } Q \\
& \widehat{y}(t, a, x)=0 \text { on } \Sigma \\
& \hat{y}(t, 0, x) \\
& \quad=e^{-\lambda_{0} t}\left(F\left(\int_{0}^{A} \beta y(\lambda, \tau) d a\right)-F\left(\int_{0}^{A} \beta y_{0} d a\right)\right) \\
& \hat{y}(0, a, x)=\tau \widehat{y}^{0}(a, x) .
\end{aligned}
$$

Multiplying (49) by $\hat{y}$ and integrating by parts, we obtain

$$
\begin{aligned}
& \frac{1}{2} \int_{0}^{A} \int_{\Omega} \hat{y}^{2}(T, a, x) d x d t \\
& \quad+\frac{1}{2} \int_{0}^{T} \int_{\Omega} \hat{y}^{2}(t, A, x) d x d a+\int_{Q}|\nabla \hat{y}|^{2} d x d a d t \\
& \quad+\int_{Q}\left(\mu+\lambda_{0}\right) \hat{y}^{2} d x d a d t \\
& =\frac{1}{2} \int_{0}^{A} \int_{\Omega} \tau^{2}\left|\hat{y}^{0}\right|^{2} d x d a \\
& \quad+\frac{1}{2} \int_{0}^{T} \int_{\Omega} \hat{y}^{2}(t, 0, x) d x d t .
\end{aligned}
$$

We have

$$
\begin{aligned}
& |\widehat{y}(t, 0, x)| \\
& \quad=e^{-\lambda_{0} t}\left|F\left(\int_{0}^{A} \beta y(\lambda, \tau) d a\right)-F\left(\int_{0}^{A} \beta y_{0} d a\right)\right| .
\end{aligned}
$$

This implies

$$
|\widehat{y}(t, 0, x)|^{2} \leq K\left|\int_{0}^{A} \beta \widehat{y}\right|^{2}
$$

then

$$
\int_{0}^{T} \int_{\Omega} \widehat{y}^{2}(t, 0, x) d x d t \leq K\|\beta\|_{\infty}^{2}\|\hat{y}\|_{L^{2}(\mathrm{Q})}^{2} .
$$

Choosing $\lambda_{0}=1+K\|\beta\|_{\infty}^{2}$ and using equality (51) we obtain

$$
\|\nabla \hat{y}\|_{L^{2}(Q)}^{2}+\|\hat{y}\|_{L^{2}(Q)}^{2} \leq C \tau^{2} \text {. }
$$

This gives

$$
y(\lambda, \tau) \longrightarrow y_{0} \quad \text { a.e on } Q \text { uniformly }
$$

and then

$$
\int_{0}^{A} \beta y(\lambda, \tau) d a \longrightarrow \int_{0}^{A} \beta y_{0} d a
$$

a.e on $Q_{T}$ uniformly.

Now we consider

$$
\begin{aligned}
& \frac{\partial z}{\partial t}+\frac{\partial z}{\partial a}-\Delta z+\left(\mu+\lambda_{0}\right) z=0 \text { in } Q \\
& z(t, a, \sigma)=0 \text { on } \Sigma \\
& z(t, 0, x)=F^{\prime}\left(\int_{0}^{A} \beta y_{0} d a\right) \int_{0}^{A} \beta e^{-\lambda_{0} t} z d a
\end{aligned}
$$

in $(0, T) \times \Omega$

$z(0, a, x)=\widehat{y}^{0}(a, x) \quad$ in $(0, A) \times \Omega$ 
$z_{\tau}=\hat{y} / \tau$ and $p_{\tau}=z_{\tau}-z$ solving

$$
\begin{aligned}
& \frac{\partial p_{\tau}}{\partial t}+\frac{\partial p_{\tau}}{\partial a}-\Delta p_{\tau}+\left(\mu+\lambda_{0}\right) p_{\tau}=0 \text { in } Q \\
& p_{\tau}(t, a, \sigma)=0 \text { on } \Sigma \\
& p_{\tau}(t, 0, x) \\
& =\frac{1}{\tau} e^{-\lambda_{0} t}\left(F\left(\int_{0}^{A} \beta y(\lambda, \tau) d a\right)-F\left(\int_{0}^{A} \beta y_{0} d a\right)\right) \\
& \quad-F^{\prime}\left(\int_{0}^{A} \beta y_{0} d a\right) \int_{0}^{A} e^{-\lambda_{0} t} \beta z d a
\end{aligned}
$$$$
\text { in }(0, T) \times \Omega
$$$$
p_{\tau}(0, a, x)=\hat{y}^{0}(a, x) \text { in }(0, A) \times \Omega .
$$

From the regularity of $F$ and the uniform convergence of $\tau \mapsto$ $y(\lambda, \tau)$, we have

$$
\begin{aligned}
& \forall \epsilon>0, \exists \tau_{0} \text { such that } \forall \tau<\tau_{0} \\
& \mid \frac{e^{-\lambda_{0} t}}{\tau} F\left(\int_{0}^{A} \beta y(\lambda, \tau) d a\right)-\frac{e^{-\lambda_{0} t}}{\tau} F\left(\int_{0}^{A} \beta y_{0} d a\right) \\
& \quad-F^{\prime}\left(\int_{0}^{A} \beta y_{0} d a\right) \int_{0}^{A} \beta e^{-\lambda_{0} t} z d a \mid \\
& \quad \leq C \int_{0}^{A} e^{\lambda_{0} t}\left|\beta p_{\tau}\right| d a+\epsilon \int_{0}^{A} e^{-\lambda_{0} t} \beta\left|z_{\tau}\right| d a
\end{aligned}
$$

Multiplying (59) by $p_{\tau}$ and integrating by parts, we obtain

$$
\begin{aligned}
& \frac{1}{2} \int_{0}^{A} \int_{\Omega} p_{\tau}^{2}(T, a, x) d x d t \\
& \quad+\frac{1}{2} \int_{0}^{T} \int_{\Omega} p_{\tau}^{2}(t, A, x) d x d a+\int_{Q}\left|\nabla p_{\tau}\right|^{2} d x d a d t \\
& \quad+\int_{Q}\left(\mu+\lambda_{0}\right) p_{\tau}^{2} d x d a d t \\
& =\frac{1}{2} \int_{0}^{T} \int_{\Omega} p_{\tau}^{2}(t, 0, x) d x d t
\end{aligned}
$$

Using (60), we obtain

$$
\begin{aligned}
& \frac{1}{2} \int_{0}^{A} \int_{\Omega} p_{\tau}^{2}(T, a, x) d x d t \\
& \quad+\frac{1}{2} \int_{0}^{T} \int_{\Omega} p_{\tau}^{2}(t, A, x) d x d a+\int_{Q}\left|\nabla p_{\tau}\right|^{2} d x d a d t \\
& \quad+\int_{Q}\left(\mu+\lambda_{0}\right) p_{\tau}^{2} d x d a d t \\
& \leq C\|\beta\|_{\infty}^{2} \epsilon+A\|\beta\|_{\infty}^{2}\left\|p_{\tau}\right\|_{L^{2}(Q)}^{2} .
\end{aligned}
$$

Choosing $\lambda_{0}$ such that $\lambda_{0}>1+A\|\beta\|_{\infty}^{2}$ we obtain

$$
\left\|p_{\tau}\right\|_{L^{2}\left((0, T) \times(0, A) ; H^{1}(\Omega)\right)}^{2} \leq C \epsilon .
$$

This implies $p_{\tau} \rightarrow 0$ a.e. in $L^{2}\left((0, T) \times(0, A) ; H_{0}^{1}(\Omega)\right)$ as $\tau \rightarrow 0$. Then $z_{\tau} \rightarrow z$ a.e. in $L^{2}\left((0, T) \times(0, A) ; H_{0}^{1}(\Omega)\right)$.

Therefore $\tau \mapsto e^{-\lambda_{0} t} y(\tau, \lambda)$ is differentiable at 0 . We conclude that $\tau \mapsto y(\lambda, \tau)$ is differentiable at 0 .

The proof of the differentiability of the function $\lambda \mapsto$ $y(\lambda, \tau)$ goes similarly.

Now let $h$ be some function in $L^{2}\left(Q_{\Theta}\right)$ and let $Q_{\omega}$ be an open and nonempty subset of $Q$. For a control function $v \in$ $L^{2}\left(Q_{\omega}\right)$, we define the functional

$$
\begin{aligned}
s(\lambda, \tau)= & \int_{\mathrm{Q}_{\Theta}} h y(\lambda, \tau) d x d a d t \\
& +\int_{\mathrm{Q}_{\omega}} v y(\lambda, \tau) d x d a d t .
\end{aligned}
$$

Using Proposition 2, one has

$$
\frac{\partial S}{\partial \tau}(0,0)=\int_{Q_{\Theta}} h y_{\tau} d x d a d t+\int_{Q_{\omega}} v y_{\tau} d x d a d t,
$$

where

$$
y_{\tau}=\lim _{\tau \rightarrow 0} \frac{y(0, \tau)-y(0,0)}{\tau}
$$

is the solution of the system

$$
\begin{aligned}
& \frac{\partial y_{\tau}}{\partial t}+\frac{\partial y_{\tau}}{\partial a}-\Delta y_{\tau}+\mu y_{\tau}=0 \quad \text { in } Q \\
& y_{\tau}(t, a, x)=0 \text { on } \Sigma \\
& y_{\tau}(t, 0, x)=\int_{0}^{A}\left(\beta F^{\prime}\left(\int_{0}^{A} \beta y(0,0) d a\right) y_{\tau}\right) d a \\
& \text { in }(0, T) \times \Omega \\
& y_{\tau}(0, a, x)=\hat{y}^{0}(a, x) \quad \text { in }(0, A) \times \Omega .
\end{aligned}
$$

We say that $S$ defines a sentinel for problem (47) if there exists $v$ such that $S$ is insensitive (at first order) with respect to the missing terms $\tau \widehat{y}^{0}$; this means

$$
\frac{\partial S}{\partial \tau}(0,0)=0
$$

for any $\widehat{y}^{0}$ and if the norm $\|v\|_{L^{2}\left(\mathrm{Q}_{\omega}\right)}$ of $v$ is minimal.

The functional $S$ assumed to be nonzero is an approached sentinel if, for any $\delta>0$, there exists $v \in L^{2}\left(Q_{\omega}\right)$ such that

$$
\begin{aligned}
& \left|\frac{\partial S}{\partial \tau}(0,0)\right| \\
& \quad=\left|\int_{Q_{\Theta}} h y_{\tau}(0,0) d x d a d t+\int_{Q_{\omega}} v y_{\tau}(0,0) d x d a d t\right| \\
& \quad \leq \delta^{2} .
\end{aligned}
$$

The existence of sentinel is equivalent to a controllability problem. 
Proposition 10. Let us consider the following system:

$$
\begin{aligned}
& -\frac{\partial q}{\partial t}-\frac{\partial q}{\partial a}-\Delta q+\mu q \\
& =\beta q(t, 0, x) F^{\prime}\left(\int_{0}^{A} \beta y(0,0) d a\right)+\chi_{\Theta} h+\chi_{\omega} v \\
& q(t, a, x)=0 \quad \text { on } \Sigma \\
& q(t, A, x)=0 \quad \text { in }(0, T) \times(0, A) \\
& q(T, a, x)=0 \quad \text { in }(0, A) \times \Omega,
\end{aligned}
$$

where $y_{0}$ is the solution of system (47) with $\lambda=\tau=0$. The approached sentinel condition (69) is equivalent to find $v \in L^{2}\left(Q_{\omega}\right)$ such that

$$
\|q(0, \cdot, \cdot)\|_{L^{2}((0, A) \times \Omega)} \leq \delta
$$

for any $\delta>0$. The exact sentinel condition is equivalent to finding $v \in L^{2}\left(Q_{\omega}\right)$ such that

$$
q(0, a, x)=0 \text { a.e in }(0, A) \times \Omega \text {. }
$$

Proof. Multiplying (70) by $y_{\tau}$ and integrating by parts, we obtain

$$
\begin{aligned}
\int_{0}^{A} & \int_{\Omega} q(0, a, x) \hat{y}^{0} d x d a d t \\
& =\int_{\mathrm{Q}_{\Theta}} h y_{\tau} d x d a d t+\int_{\mathrm{Q}_{\omega}} v y_{\tau} d x d a d t .
\end{aligned}
$$

And then the approached sentinel condition

$$
\left|\int_{Q}\left(\chi_{\Theta} h+\chi_{\omega} v\right) y_{\tau} d t d a d x\right| \leq \delta^{2}
$$

is equivalent to

$$
\left|\int_{0}^{A} \int_{\Omega} q(0, a, x) \hat{y}^{0} d x d a\right| \leq \delta^{2}
$$

for every

$$
\widehat{y}^{0} \in L^{2}((0, A) \times \Omega) .
$$

That is equivalent to

$$
\|q(0, a, x)\|_{L^{2}((0, A) \times \Omega)} \leq \delta .
$$

We have the following result.

Proposition 11. Let $h \in L^{2}\left(e_{M}\right)$, if $F \in C^{1}(\mathbb{R})$ and $F^{\prime} \in$ $L^{\infty}(\mathbb{R})$; then under assumptions $\left(H_{1}\right),\left(H_{2}\right)$, and $\left(H_{3}\right)$ there exists a unique control $v \in L^{2}\left(Q_{\omega}\right)$ satisfying (68).

Proof. Replacing $b(t, x)$ in (21) by $F^{\prime}\left(\int_{0}^{A} \beta y(0,0) d a\right)$ we obtain the approached sentinel, and we obtain the sentinel when $\delta$ goes to zero.

\section{Information Provided by the Sentinel}

The function

$$
y_{\lambda}=\lim _{\lambda \rightarrow 0} \frac{y(\lambda, 0)-y(0,0)}{\lambda}
$$

is the solution of the system

$$
\begin{aligned}
& \frac{\partial y_{\lambda}}{\partial t}+\frac{\partial y_{\lambda}}{\partial a}-\Delta y_{\lambda}+\mu y_{\lambda}=\widehat{\xi}(t, a, x) \\
& y_{\lambda}(t, a, x)=0 \quad \text { on } \Sigma \\
& y_{\lambda}(t, 0, x)=\int_{0}^{A}\left(\beta F^{\prime}\left(\int_{0}^{A} \beta y(0,0) d a\right) y_{\lambda}\right) d a
\end{aligned}
$$$$
\text { in }(0, T) \times \Omega
$$

$$
y_{\lambda}(0, a, x)=0 \quad \text { in }(0, A) \times \Omega .
$$

We have

$$
\begin{aligned}
S_{\mathrm{obs}}(\lambda, \tau) \simeq & S(0,0)+\lambda \frac{\partial S}{\partial \lambda}(0,0) \\
& +\tau \frac{\partial S}{\partial \tau}(0,0) \Longleftrightarrow \\
S_{\text {obs }}(\lambda, \tau)-S(0,0) \simeq & \lambda \frac{\partial S}{\partial \lambda}(0,0) .
\end{aligned}
$$

Multiplying system (22) by $y_{\lambda}$ and integrating by parts, we obtain

$$
\int_{Q} q \widehat{\xi} d x d a d t=\int_{Q}\left(\chi_{\Theta} h+\chi_{\omega} v\right) y_{\lambda} d x d a d t
$$

and then

$$
\begin{aligned}
& \int_{Q} \lambda q \hat{\xi} d x d a d t \\
& \quad=\int_{Q}\left(\chi_{\Theta} h+\chi_{\omega} v\right)\left(m_{0}-y(0,0)\right) d x d a d t .
\end{aligned}
$$

Knowing $h, v, m_{0}$, and $y(0,0), q$ can be calculated. Thus we obtain an integral equation in $\lambda \hat{\xi}$.

\section{Conflict of Interests}

The authors declare that there is no conflict of interests regarding the publication of this paper.

\section{Acknowledgment}

The first author thanks AIMS South Africa for its partial financial support and for hospitality.

\section{References}

[1] A. Fursikov and O. Y. Imanuvilov, Controllability of Evolution Equations, Lecture Notes, Research Institute of Mathematics, Seoul National University, Seoul, Republic of Korea, 1996. 
[2] O. Traore, "Null controllability of a nonlinear population dynamics problem," International Journal of Mathematics and Mathematical Sciences, vol. 2006, Article ID 49279, 20 pages, 2006.

[3] B. Ainseba and M. Langlais, "On a population dynamics control problem with age dependence and spatial structure," Journal of Mathematical Analysis and Applications, vol. 248, no. 2, pp. 455474, 2000.

[4] J. Klamka, "Schauder's fixed-point theorem in nonlinear controllability problems," Control and Cybernetics, vol. 29, no. 1, pp. 153-165, 2000.

[5] J. Klamka and A. Świerniak, "Controllability of a model of combined anticancer therapy," Control and Cybernetics, vol. 42, no. 1, pp. 123-138, 2013.

[6] J. L. Lions, Sentinelle pour les Systèmes Distribués à Données Incomplètes, vol. 21, Masson, RMA, Paris, France, 1992.

[7] O. Bodart, Application de la méthode des sentinelles à l'identification de sources de pollution dans un système distribué [Ph.D. thesis], Université de Technologie Compiègne, 1994.

[8] O. Bodart and C. Fabre, "Controls insensitizing the norm of the solution of a semilinear heat equation," Journal of Mathematical Analysis and Applications, vol. 195, no. 3, pp. 658-683, 1995.

[9] O. Bodart and C. Fabre, "Contrôle insensibilisant la norme de la solution d'une équation de la chaleur semi-linéaire," Comptes Rendus de l'Académie des Sciences. Série I. Mathématique, vol. 316, no. 8, pp. 789-794, 1993.

[10] O. Bodart and P. Demeestere, "Sentinels for the identification of an unknown boundary," Mathematical Models and Methods in Applied Sciences, vol. 7, no. 6, pp. 871-885, 1997.

[11] P. Demeestere, Methode de sentinelles: etude comparative et application a l'identification d'une frontiere. Controle du temps d'explosion d'une equation de com-bution [Ph.D. thesis], University of Technology of Compiègne, Compiègne, France, 1997.

[12] J. P. Kernevez, The Sentinel Method and Its Application to Environmental Pollution Problems, CRC Press, Boca Raton, Fla, USA, 1997.

[13] S. Somdouda, "Parameters identification in population dynamics problem," African Diaspora Journal of Mathematics, vol. 13, no. 2, pp. 81-99, 2012. 


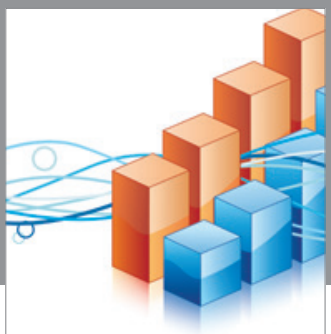

Advances in

Operations Research

vatem alat4

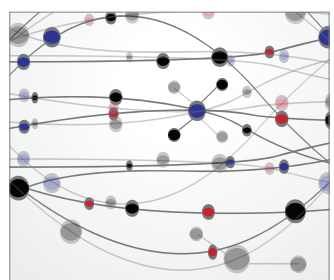

\section{The Scientific} World Journal
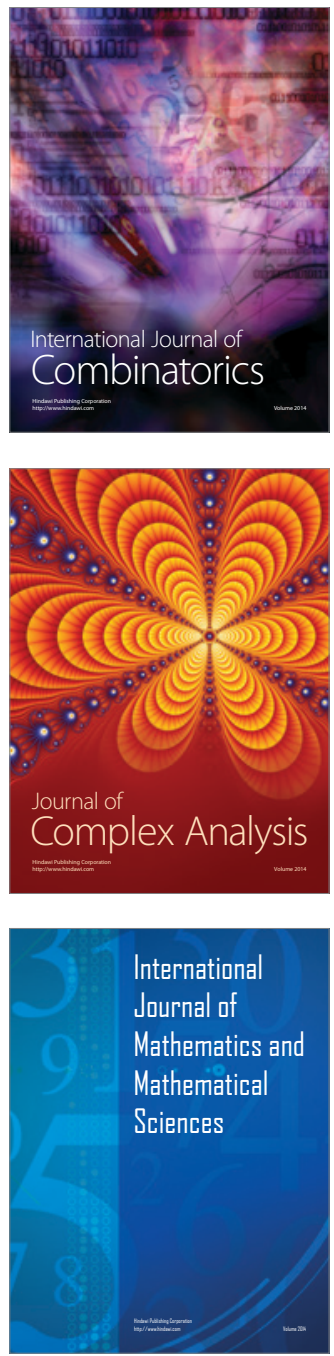
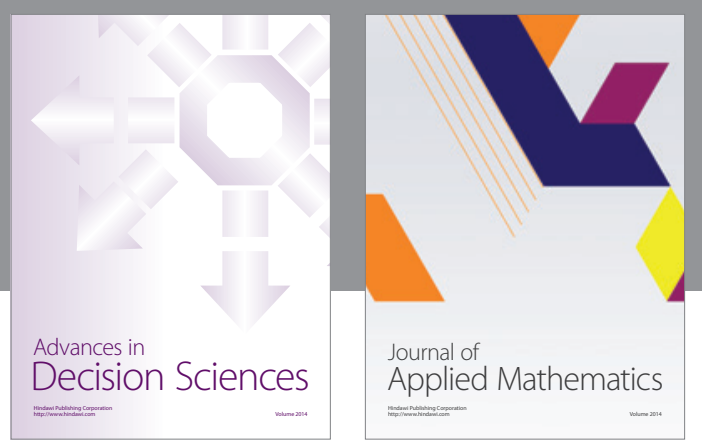

Algebra

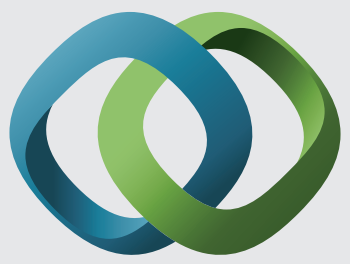

\section{Hindawi}

Submit your manuscripts at

http://www.hindawi.com
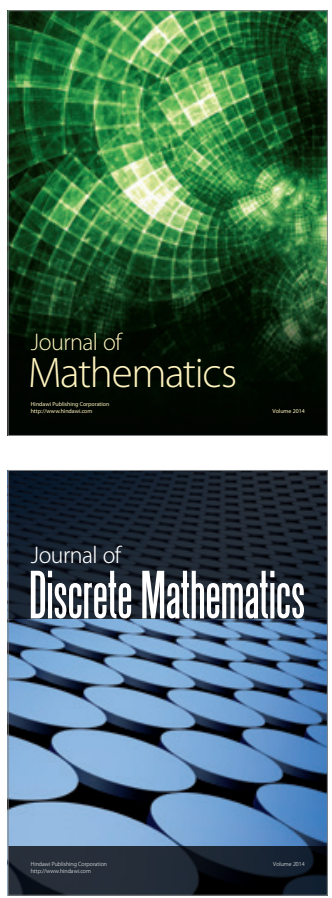

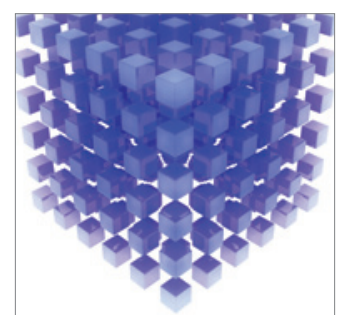

Mathematical Problems in Engineering
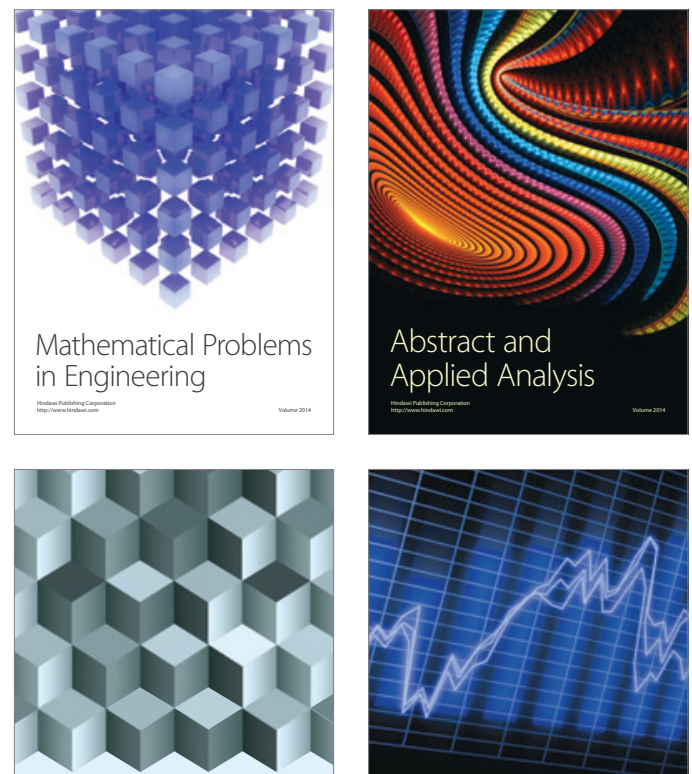

Journal of

Function Spaces

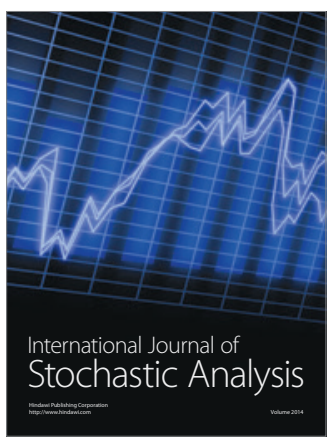

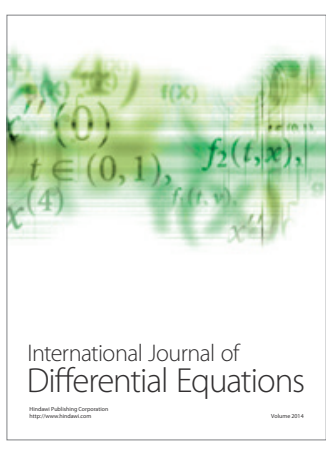
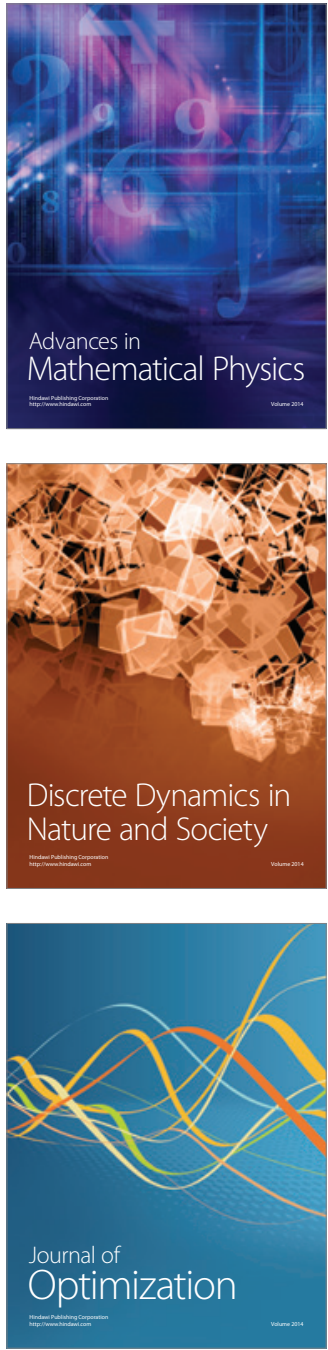